\title{
Magnetophoretic induction of curvature in coleoptiles and hypocotyls ${ }^{1}$
}

\author{
Oleg A. Kuznetsov and Karl H. Hasenstein ${ }^{2}$ \\ Biology Department, University of Southwestern Louisiana, Lafayette, LA 70504-2451, USA
}

Received 19 February 1997; Accepted 14 July 1997

\begin{abstract}
Coleoptiles of barley (Hordeum vulgare) were positioned in a high gradient magnetic field (HGMF, dynamic factor $\nabla \mathrm{H}^{2} / 2$ of $\left.10^{9}-10^{10} \mathrm{Oe}^{2} \mathrm{~cm}^{-1}\right)$, generated by a ferromagnetic wedge in a uniform magnetic field) and rotated on a $1 \mathrm{rpm}$ clinostat. After $4 \mathrm{~h} 90 \%$ of coleoptiles had curved toward the HGMF. The cells affected by HGMF showed clear intracellular displacement of amyloplasts. Coleoptiles in a magnetic field next to a non-ferromagnetic wedge showed no preferential curvature. The small size of the area of nonuniformity of the HGMF allowed mapping of the sensitivity of the coleoptiles by varying the initial position of the wedge relative to the coleoptile apex. When the ferromagnetic wedge was placed $1 \mathrm{~mm}$ below the coleoptile tip only $58 \%$ of the coleoptiles curved toward the wedge indicating that the cells most sensitive to intracellular displacement of amyloplasts and thus gravity sensing are confined to the top $1 \mathrm{~mm}$ portion of barley coleoptiles. Similar experiments with tomato hypocotyls (Lycopersicum esculentum) also resulted in curvature toward the HGMF. The data strongly support the amyloplast-based gravity-sensing system in higher plants and the usefulness of HGMF to substitute gravity in shoots.
\end{abstract}

Key words: Avena sativa, Hordeum vulgare, Lycopersicon esculentum, curvature, gravitropism, high gradient magnetic field, magnetophoresis.

\section{Introduction}

The primary act of gravity sensing in higher plants is presumed to be the displacement of amyloplasts inside receptor cells, the statocytes. Inside these statocytes dense amyloplasts sediment due to the gravity force and trigger complex events that result in the reorientation of plant organs relative to their normal orientation in the gravity field. This gravitropic curvature of all or part of the organ(s) re-establishes the so-called gravitropic set point angle (Digby and Firn, 1995).

Previous work has shown that high gradient magnetic fields (HGMF) exert ponderomotive forces on amyloplasts that are proportional to the dynamic factor of the field $\nabla \mathrm{H}^{2} / 2$, and opposite to the field gradient. Since starch-filled amyloplasts are more diamagnetic than the aqueous cytoplasm they move away from the denser magnetic field (Audus, 1960; Audus and Whish, 1964; Kuznetsov and Hasenstein, 1996; Kuznetsov and Kuznetsov, 1989; Piruzyan et al., 1980). Therefore, HGMF with a dynamic factor $\nabla \mathrm{H}^{2} / 2$ of $c .10^{9}-10^{10} \mathrm{Oe}^{2}$ $\mathrm{cm}^{-1}$ should be able to mimic the directional effect of gravity. Earlier work with roots of flax (Linum usitatissimum) and Arabidopsis showed starch-dependent curvature away from the stronger field areas, i.e. away from the edge of a magnetized ferromagnetic wedge or away from the depth of the gap between permanent magnets (Kuznetsov and Hasenstein, 1996). Roots of starchless mutants of Arabidopsis did not respond to the HGMF, which implies that the relatively strong diamagnetic starch is necessary for the magnetophoretic displacement of amyloplasts.

For amyloplast-based plant statocytes, the magnetic gradient leads to a polarity such that the stronger field area is comparable to the 'up', and the weaker field area analogous to the 'down' direction of the gravity vector. Positively gravitropic roots curve parallel to the gravity vector. Therefore, roots are expected to curve away from the stronger field area. In contrast, shoots typically show negative gravitropism and curve anti-parallel to the

\footnotetext{
1 Dedicated to Professor LJ Audus.

${ }^{2}$ To whom correspondence should be addressed. Fax: +1 318482 5834. E-mail: hasenstein@usl.edu

Abbreviations: HGMF, high gradient magnetic field.
} 
gravity vector. In a HGMF they should curve toward the stronger field area.

Earlier experiments attempted to induce gravitropiclike curvature in shoots of Lepidium and sporangia of Phycomyces (Schwarzacher and Audus, 1973). However, a relatively large area of non-uniform magnetic field resulted in a relatively weak field gradient $\left(10^{3}-10^{4} \mathrm{Oe}^{2}\right.$ $\mathrm{cm}^{-1}$ ) and the dynamic factor $\nabla \mathrm{H}^{2} / 2$ did not exceed $4.5 \times 10^{7} \mathrm{Oe}^{2} \mathrm{~cm}^{-1}$. Under such conditions the amyloplasts are subjected to ponderomotive forces that are about 1/100 of the gravity force and Avena coleoptiles curved away from the stronger field areas. Because of these results, Schwarzacher and Audus (1973) concluded that HGMF did not substitute gravity and that the 'magnetotropism' causes physiological responses different from reorientation in the gravity field. In this paper the usefulness of magnetophoretic amyloplast displacement for the study of the gravisensing mechanism of shoots is re-examined and it is shown that the ponderomotive force generated by a sufficiently strong HGMF can exert the same effect as gravity. If the dynamic factor $\nabla \mathrm{H}^{2} / 2$ of in the order of $10^{9}-10^{10} \mathrm{Oe}^{2} \mathrm{~cm}^{-1}$, the effective ponderomotive forces acting on amyloplasts are approximately equal to gravity and should exert corresponding effects such as the displacement of amyloplasts.

\section{Materials and methods}

\section{Plant material}

Seeds of barley (Hordeum vulgare cv. Himalaya), oat (Avena sativa cv. Victory), or tomato (Lycopersicon esculentum cv. Ailsa Craig) were germinated on moist filter paper in darkness at about $25^{\circ} \mathrm{C}$. After $1.5-2 \mathrm{~d}$, the germinating seeds were placed upright in holders prepared from $2.5 \mathrm{~cm}$ sections of $1 \mathrm{ml}$ pipette tips. The conical part was cut to an inner diameter of about 3 $\mathrm{mm}$ and the bottom portions were filled with moist soil, and sealed with Parafilm. The holders with seedlings were placed vertically in a dark cabinet for c. $24 \mathrm{~h}$ at room temperature. Then straight shoots protruding $3-8 \mathrm{~mm}$ above the orifice of the holder were used for experiments.

\section{Magnetic system}

A HGMF was generated using a ferromagnetic wedge (dihedral angle of $92^{\circ}$, height $1.8 \mathrm{~mm}$, length $25 \mathrm{~mm}$ ). This wedge was coated with plastic to avoid corrosion and possible chemical influence of metal ions on plant curvature (Hasenstein et al., 1988). The wedge was magnetized by a uniform magnetic field ( $4.5 \mathrm{kOe})$ generated between two permanent magnets $\left(\mathrm{SmCo}_{5}\right.$, $40 \times 40 \times 16 \mathrm{~mm}$ each). The magnetic field near the edge of the wedge is stronger than the field between the magnets, and the gradient $\nabla H$ is directed toward the wedge (Fig. 1). Diamagnetic substances (magnetic susceptibility $\chi<0$ ), such as starch-filled amyloplasts, experience a force that is directed away from the edge of the wedge.

The area where the ponderomotive force affecting the amyloplasts is equal to or greater than $1 \mathrm{~g}$ was about $0.3 \mathrm{~mm}$ along the wedge edge (shaded area in Fig. 2). If a tip of a coleoptile is positioned in close proximity to the centre of the wedge edge there will be no net field gradient parallel to the

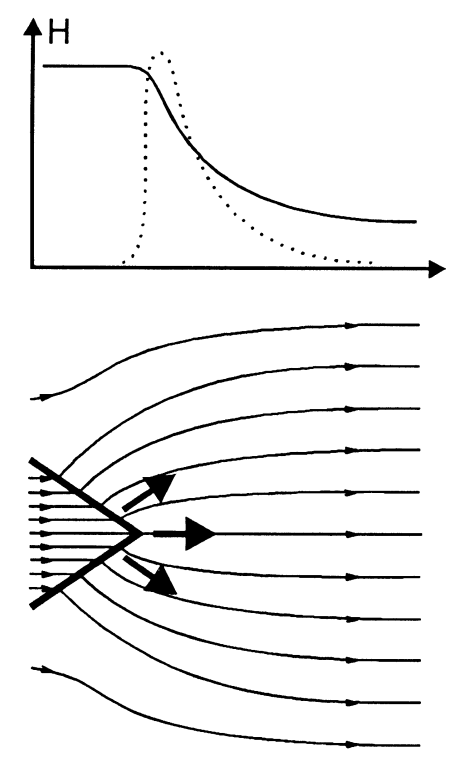

Fig. 1. Magnetic field near a ferromagnetic wedge magnetized by a uniform external magnetic field. The density of field lines is proportional to the field intensity (solid line in upper diagram) and increases toward the wedge tip, such that the gradient of the field (dotted line) is directed toward the tip. The arrows indicate the direction of the force acting on amyloplasts (modified from Kuznetsov and Hasenstein, 1996).

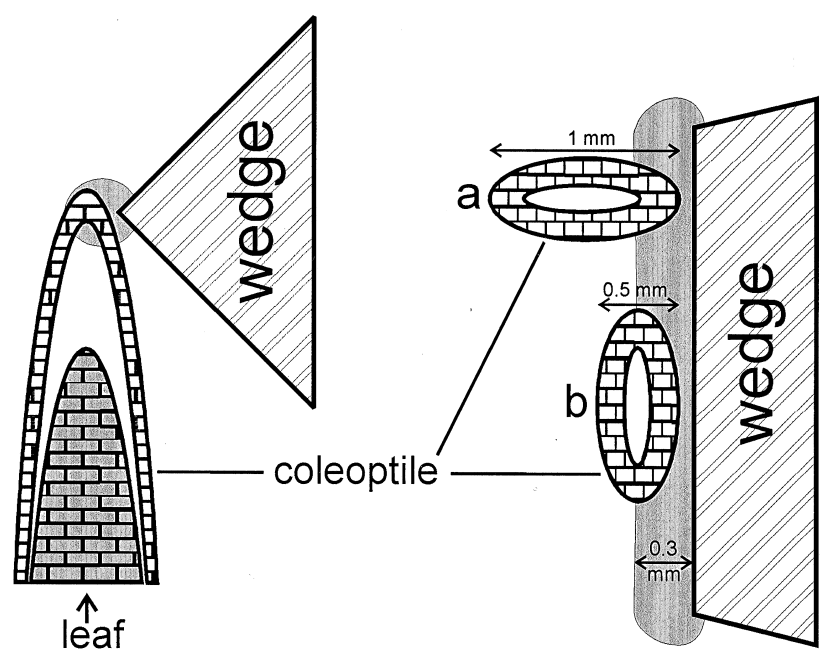

Fig. 2. Mutual positioning of coleoptiles and a ferromagnetic wedge, magnetized by a uniform magnetic field as viewed from the side (left), and top (right). The shaded zone represents the area of the HGMF, where the ponderomotive forces affecting starch-filled amyloplasts are equal to or greater than the force of gravity. The relative orientation between the long axis of the coleoptile and wedge was either perpendicular (a) or parallel (b).

edge of the wedge and the coleoptile should curve toward the wedge.

Amyloplasts that are within $0.3 \mathrm{~mm}$ of the wedge edge experience a magnetic force comparable to gravity. Since barley coleoptiles have an elliptical cross-section near the tip (about $0.5 \times 1 \mathrm{~mm}$ diameter) and since a distance of $c .0 .1 \mathrm{~mm}$ was maintained between wedge and coleoptile or hypocotyl to avoid touch-induced reactions, part of the coleoptile amyloplasts experienced a considerably reduced force, less than $10^{-1} \mathrm{~g}$. 
Therefore, the ponderomotive forces acting on amyloplasts equal to gravity were confined to a small area of the plant tissue.

Pipette tips with seedlings were attached to a holder that could be moved perpendicular to the wedge allowing precise positioning of the wedge relative to the seedling. In all experiments, the wedge edge was placed not farther than 0.1 $\mathrm{mm}$ from the surface of the coleoptile and care was taken to avoid touching (Jaffe, 1973; Braam and Davis, 1990). The positioning of the wedge relative to the tip of the coleoptile or hypocotyl permitted the analysis of the longitudinal distribution of gravisensitivity. For coleoptiles, four positions were examined; the wedge was placed $0.5 \mathrm{~mm}$ above, even with, or 1 or $3 \mathrm{~mm}$ below the tip.

To minimize the influence of gravity, the system was mounted on a $1 \mathrm{rpm}$ clinostat. The axis of the coleoptile was perpendicular to the horizontal axis of rotation of the clinostat. The entire set-up was placed in a dark cabinet for 10-15 min before clinorotation was started.

After $4 \mathrm{~h}$ of rotation the direction of curvature was evaluated. When the coleoptile tip or hypocotyl hook was displaced $1 \mathrm{~mm}$ or more from the longitudinal axis or when a distinct kink appeared the specimen was counted as curved.

The distributions of shoot curvature (growth parallel to the wedge edge, curvature toward, both considered 'positive', no curvature, or curvature away from the wedge) after different treatments were compared using non-parametric, two-tailed Kolmogorov-Smirnov tests (Conover, 1971; SysStat Software, 1992).

\section{Microscopy}

Coleoptiles fixed on the clinostat under HGMF, gravistimulation or in vertical orientation (without HGMF, controls) in $4 \%$ formaldehyde (in phosphate buffer, $\mathrm{pH} 6.2$, containing 5\% DMSO). Then, the apical $5 \mathrm{~mm}$ were cut and incubated in fixative for an additional $2 \mathrm{~h}$. After dehydration in ethanol, acetone and embedding in Spurr's resin, sections $(2 \mu \mathrm{m})$ were stained $(0.5 \%$ methylene blue/borax, $\mathrm{pH} 9.5)$. The sections were examined under a microscope and photographed.

\section{Results and discussion}

The curvature of most coleoptiles occurred either toward or parallel to the wedge. If the magnetized wedge was located next to the coleoptile tips before the onset of clinorotation, most coleoptiles curved toward the wedge (Figs 3,4) and stayed essentially in a plane perpendicular to the wedge edge. In some cases coleoptiles curved initially toward the wedge but also curved parallel to the wedge edge. When the coleoptiles were positionend near the ferromagnetic wedge $90 \%$ curved toward the HGMF. Thus barley coleoptiles, subjected to a sufficiently strong HGMF, behaved as if graviresponding to a force directed away from the wedge edge.

Curvature toward the wedge or initial curvature toward the wedge with subsequent growth parallel to the wedge is consistent with the orientation of the HGMF. If the initial growth direction of the coleoptile is perpendicular to the wedge edge, there will be no net lateral component of the field gradient and the coleoptile should curve toward the wedge while continuing to grow perpendicular to the wedge edge. The tip will eventually grow out of the HGMF area, ultimately producing a well-defined arch of the coleoptile around the wedge (Fig. 3). However, if the coleoptile does not grow strictly perpendicular to the wedge or undergoes nutational movements, the HGMF will have a lateral component that can affect the coleoptile to curve toward the strongest gradient, i.e. the edge of the wedge. Such gradient may induce curvature toward and eventually growth parallel to the wedge. Once the coleoptile has established an orientation parallel to the wedge, there will always be a lateral force directing a positively graviresponsive organ toward the wedge, thus keeping the coleoptile parallel to the wedge. Initial instability and deviation from the plane perpendicular to the wedge could be caused by imperfect positioning or
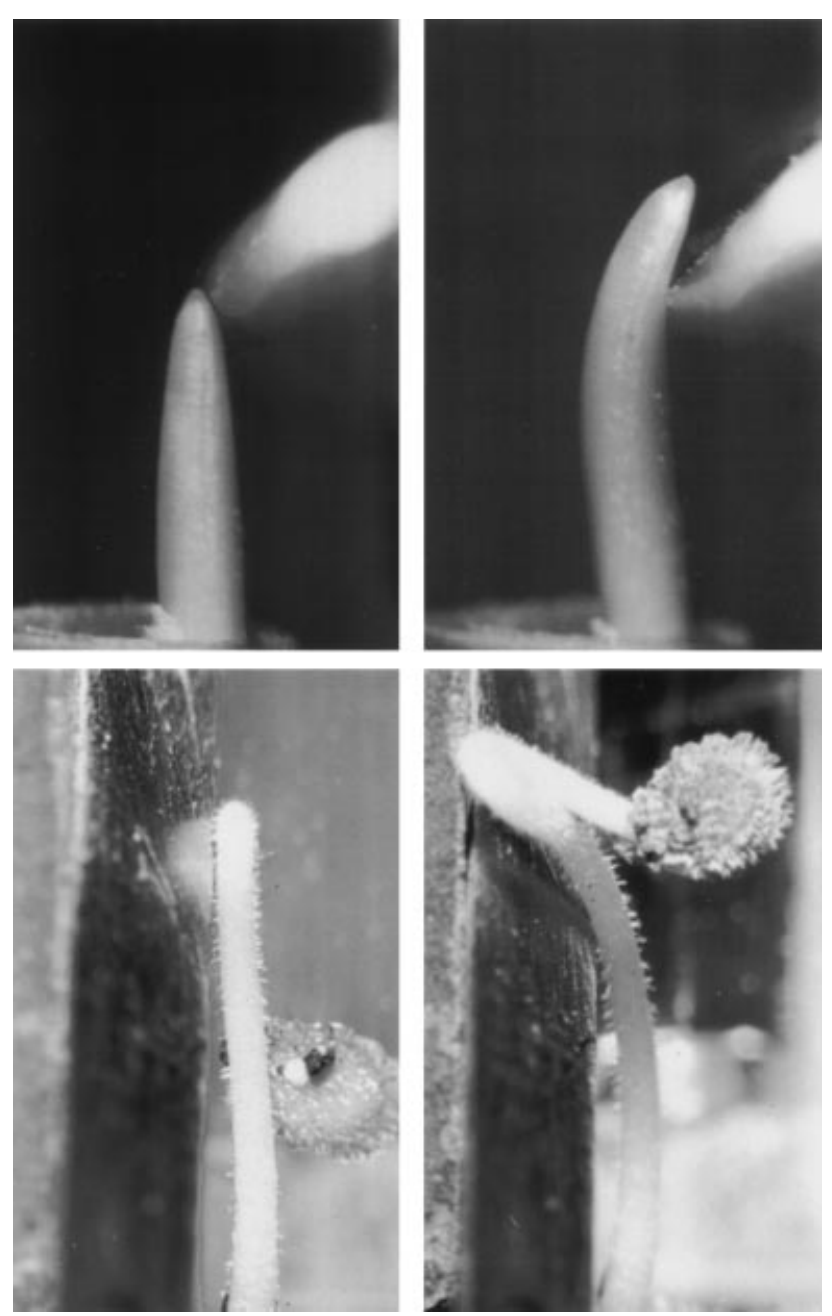

Fig. 3. Induction of curvature by high gradient magnetic field in barley coleoptiles (top) and etiolated tomato seedlings (bottom). Curvature was initiated by positioning a ferromagnetic wedge close to the coleoptile tip or hypocotyl hook. The seedlings, wedge, and magnets (not shown) were mounted on a $1 \mathrm{rpm}$ clinostat with the coleoptile or hypocotyl growing perpendicular to the horizontal axis of rotation. Presumably due to the action of an HGMF on amyloplasts the shoots curved toward and around the wedge as if responding negatively gravitropic. The images on the right show curvature after $5 \mathrm{~h}$ of clinorotation. 
by nutational movements, possibly augmented by clinorotation.

For control experiments a (non-ferromagnetic) brass wedge of the same size and shape and coating as the ferromagnetic wedge was placed between black plastic blocks of the same size as the magnets. Positioning was identical to the experiments with a ferromagnetic wedge and the set-up was mounted on the same clinostat. Under those conditions there was no net curvature. About 20\% of the coleoptiles curved toward the brass wedge, most coleoptiles showed no curvature $(45 \%)$ and $35 \%$ curved away from the wedge (Fig. 4). This distribution of curvature is different from that in the HGMF $(P<0.001)$. The tendency to curve is probably enhanced due to clinorotation, but there was no preferential curvature away or toward the brass wedge.

In different experiments seedlings were placed between plastic blocks on a clinostat without wedge to determine if the deviation from a uniform distribution of curvature might be caused by touch-induced reactions and/or clinorotation. The resulting distribution of curvature was similar and the distribution of curvature with and without wedge in a set-up without magnets was not significantly different $(P>0.95$, Fig. 4).

The possible effect of the uniform magnetic field on coleoptile curvature was investigated by placing seedlings

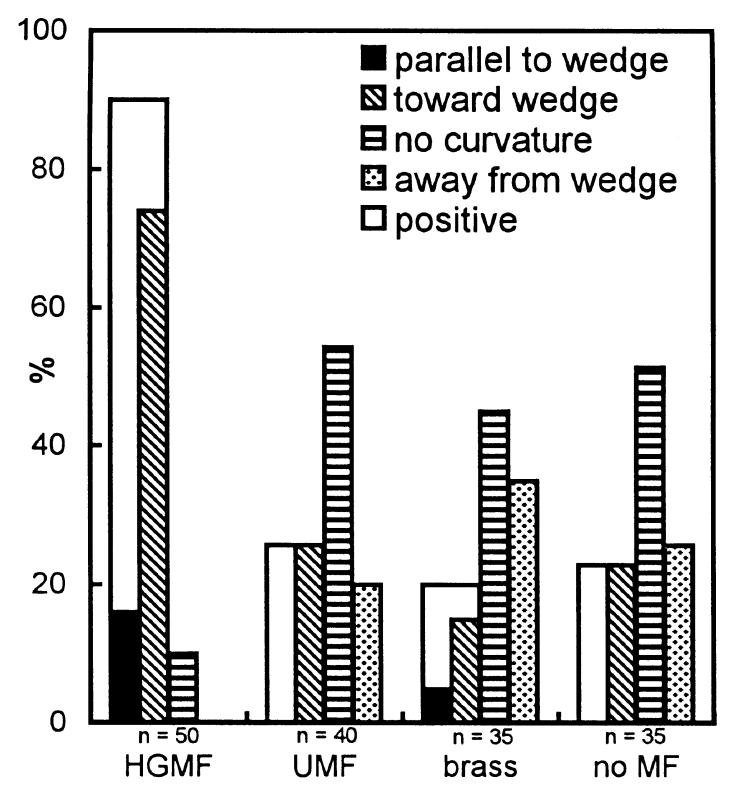

Fig. 4. Distribution of curvature of 3-d-old barley coleoptiles after placing the coleoptiles inside a uniform magnetic field $(4.5 \mathrm{kOe})$ in close proximity to an edge of a ferromagnetic wedge (HGMF), a uniform field without a wedge (UMF) or a non-ferromagnetic wedge of equal size (brass). Controls were observed without a magnetic field (no MF). Coleoptiles and, if applicable, wedges were mounted on a clinostat with the coleoptile axis perpendicular to the horizontal axis of rotation and rotated at $1 \mathrm{rpm}$. Coleoptiles curvature was evaluated after $4 \mathrm{~h}$ and considered curved when the angle exceeded $10^{\circ}$. Coleoptiles growing toward or parallel to the wedge are combined (open bar) to represent 'positive curvature'. between the same magnets but without wedge. The distribution of curvature was statistically similar to that without magnetic field $(P<0.001)$, indicating that a uniform magnetic field of about $4.5 \mathrm{kOe}$ does not influence curvature of barley coleoptiles.

Similar to previous results (Kuznetsov and Hasenstein, 1996), the curvature of coleoptiles under HGMF was accompanied by the displacement of amyloplasts similar to gravistimulated coleoptiles (Fig. 5). Vertically oriented coleoptiles show relatively uniform distribution of basally sedimented amyloplasts (Fig. 5A), but both magnetophoretically stimulated (Fig. 5B) and gravistimulated (Fig. 5C) coleoptiles display laterally distributed amyloplasts. This result suggests that curvature is initiated by amyloplast displacement either by a ponderomotive force or gravity and supports amyloplasts as the primary site of graviperception.

Substituting barley with oat seedlings, the species used by Schwarzacher and Audus (1973), also resulted in curvature of coleoptiles toward the stronger field (Fig. 6 ), and distribution of the curvature response was similar to that of barley $(P \geq 0.95)$. Similarly, experiments with tomato seedlings also showed curvature of shoots toward stronger field area (Fig. 6). Therefore the observed reaction is not species-specific.

If the wedge edge is not perpendicular to the coleoptile axis, amyloplasts in such a coleoptile experience a lateral component of the ponderomotive magnetic force. The smaller the angle between the wedge edge and the coleoptile axis, the stronger such a lateral component. The lateral force component should cause the coleoptile to curve parallel to the wedge. As the coleoptile is curving toward the wedge edge, the angle between the coleoptile and the edge gets smaller, which leads to an increase in the lateral components of the ponderomotive force. This would cause the coleoptile to continue to grow along the edge. Placing the wedge initially at a $45^{\circ}$ angle with the coleoptile axis, the number of seedlings, growing parallel to the edge increased dramatically (from 16-70\%, Fig. 6), and overall percentage of curvature toward the stronger field increased, presumably due to an increased volume of plant tissue affected by HGMF and the longer time it spent in the HGMF. Reduction of the angle to $10^{\circ}$ increased parallel growth further (Fig. 7).

Starch containing organelles are found in long files of cells in the terminal $7-11 \mathrm{~mm}$ portion of barley coleoptiles. The ponderomotive force that affects amyloplasts is greater than or equal to gravity for about $0.3 \mathrm{~mm}$ from the wedge (Fig. 2). The effective volume of the HGMF is much smaller than that in which cells with amyloplasts are found and, therefore, it is possible to stimulate a subset of cells along the coleoptile apex. Positioning the coleoptile tip at the beginning of the experiment $0.5 \mathrm{~mm}$ below, even with, or 1 or $3 \mathrm{~mm}$ above the wedge edge should reveal the sensitivity profile of the coleoptile tip. 


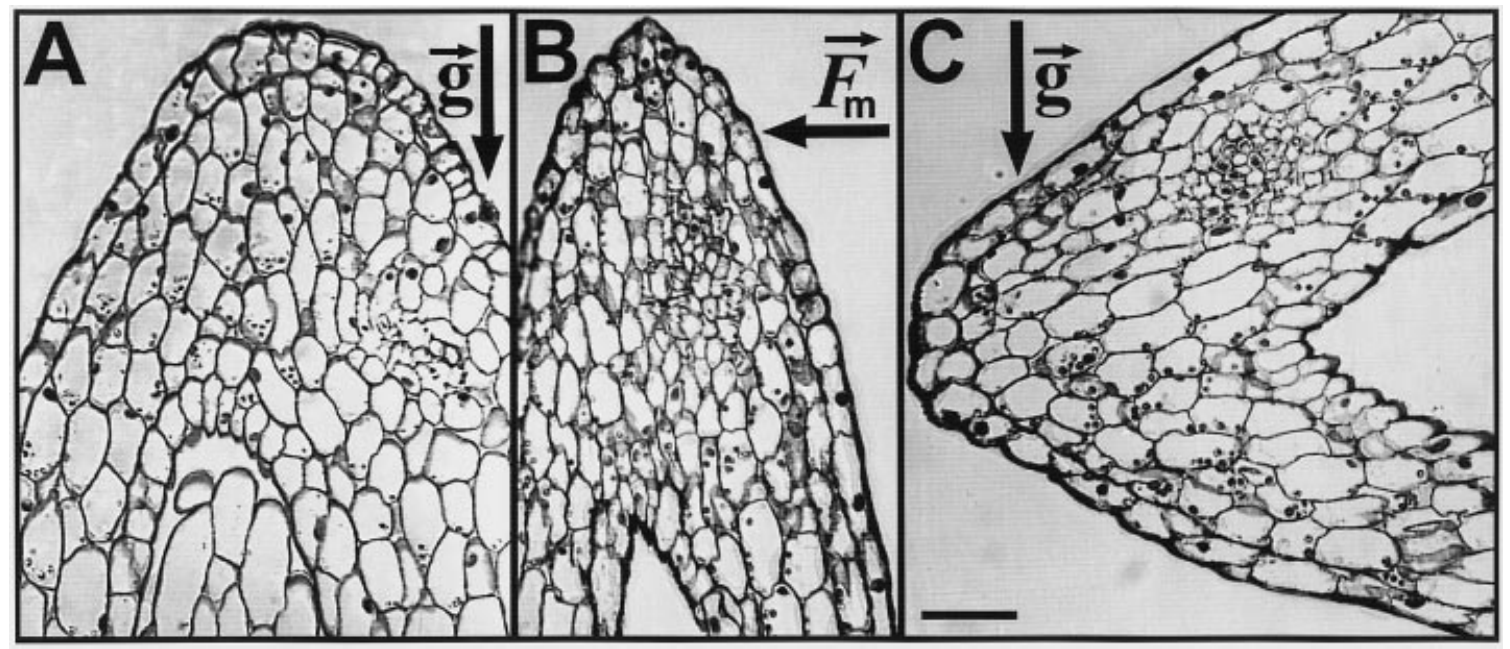

Fig. 5. Micrographs of amyloplast distribution in barley coleoptiles. Vertically oriented coleoptiles show basally sedimented amyloplasts (A). The magnetophoretically stimulated coleoptile clearly shows displacements of amyloplasts away from the HGMF in response to the magnetic force (B, cut parallel to the smaller diameter of the coleoptile). Gravistimulated coleoptiles display laterally sedimented amyloplasts (C). Bar $=50 \mu \mathrm{m}$, same magnification in all images.

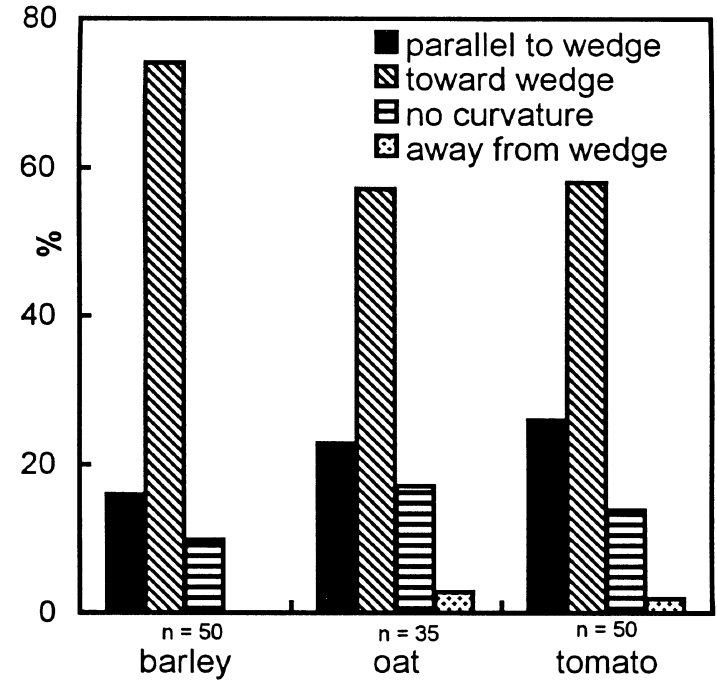

Fig. 6. Curvature of barley (Hordeum vulgare) and oat (Avena sativa) coleoptiles, and tomato (Lycopersicon esculentum) hypocotyls due to ponderomotive forces in a high gradient magnetic field.

Different initial positions of the wedge and coleoptile resulted in different curvature (Fig. 8). When the wedge was placed $0.5 \mathrm{~mm}$ above the tip, fewer coleoptiles curved than when the wedge and tip were precisely aligned. The reduced curvature toward the wedge was compensated by a higher percentage of coleoptiles growing parallel to the wedge. Due to the elongation growth that moved the coleoptile tip into the effective area of the HGMF, the mutual positioning of wedge and coleoptile tip may not have been as precise or efficient as when the tip was aligned with the wedge edge. When the coleoptile tip was initially placed below the edge of the wedge the chance for nutational movements and thus a net component of

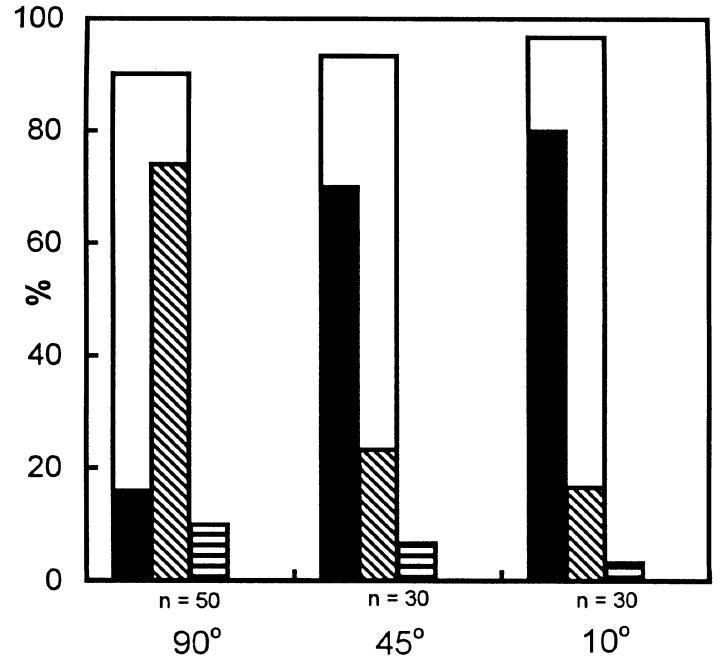

Fig. 7. Curvature of barley coleoptiles to a high gradient magnetic field generated by a ferromagnetic wedge. The wedge was positioned at $90^{\circ}$, $45^{\circ}$, or $10^{\circ}$ angle to the coleoptile axis. Coleoptile growth parallel to the wedge (black bars) compared with perpendicular curvature (diagonally hatched bars) increased as the angle between wedge and coleoptile decreased. The total 'positive response' (sum of curvature toward and parallel to wedge, open bars) also increased, presumably due to longer exposure to the HGMF (horizontally striped bars indicate no curvature).

the ponderomotive force lateral to the coleoptiles may have caused curvature that resulted in growth parallel to the wedge. When both responses (curvature toward and growth parallel to the wedge edge) are considered positive, the response patterns for the two initial positions were very similar (Fig. 8).

There was a significant reduction in the percentage of coleoptiles curving toward the wedge when it was positioned below the coleoptile tip (Fig. 8). When the wedge 


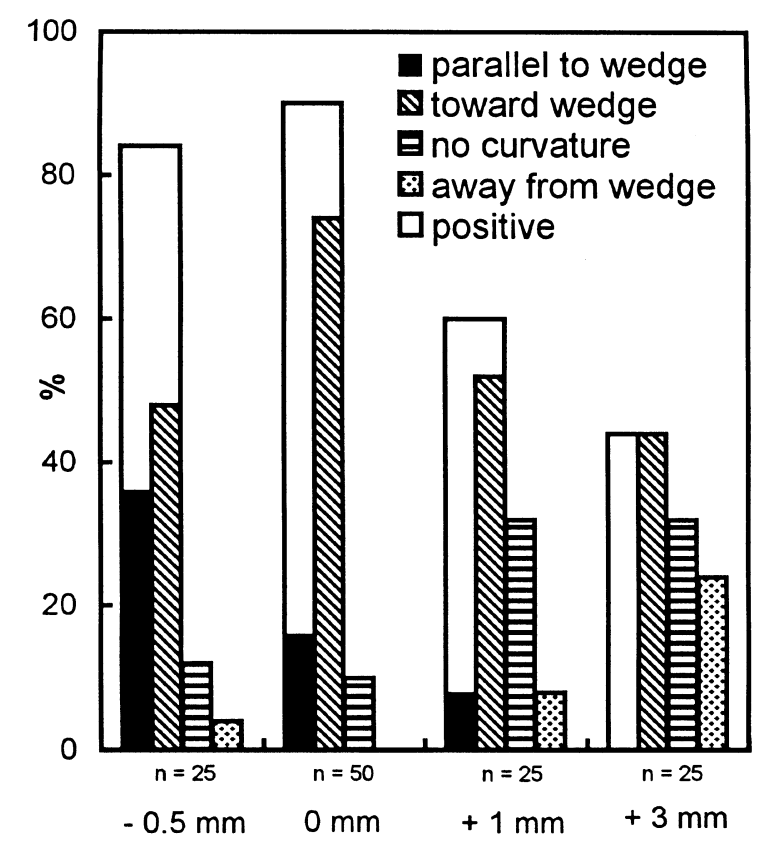

Fig. 8. Curvature of 3-d-old barley coleoptiles with initial position of the coleoptile tip $0.5 \mathrm{~mm}$ below, even with, or 1 or $3 \mathrm{~mm}$ above a magnetized ferromagnetic wedge. Coleoptiles growing toward or parallel to the wedge are combined as 'positive curvature' (open bar).

was positioned $3 \mathrm{~mm}$ below the tip the curvature was similar to that obtained with a non-ferromagnetic wedge and not different from non-stimulated coleoptiles (Figs 4, 9).

These results suggest that the cells most sensitive to intracellular displacement of amyloplasts and thus gravity sensing, are confined to the top $1 \mathrm{~mm}$ portion of barley coleoptiles. However, gravisensitivity is not limited to this area because coleoptiles from which the terminal mm was

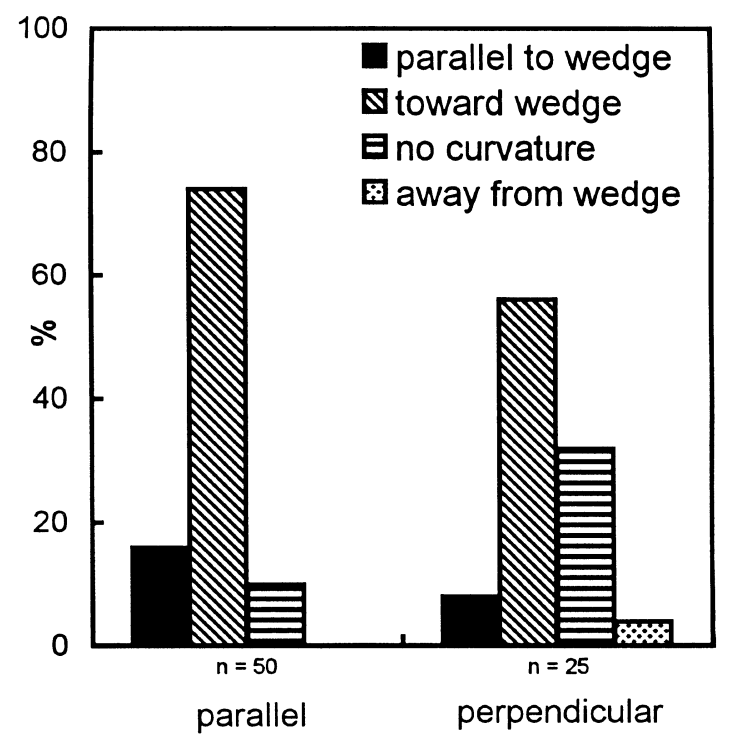

Fig. 9. Curvature of barley coleoptiles when the long diameter was oriented parallel or perpendicular (see Fig. 2) to the wedge. removed, still curved upward if placed horizontally. However, the response is less strong than that of intact coleoptiles (Table 1). The reduced graviresponse suggests that statocytes within the apical portion generate the strongest response to amyloplasts displacement.

The geometry of the tissue further affects the number of amyloplasts that experience a ponderomotive force comparable to gravity. Three $\mathrm{mm}$ below the tip the coleoptile has an almost cylindrical shape with a diameter of 1.5-2 mm. Closer to the tip the cross-sectional area is elliptic with 0.5 and $1 \mathrm{~mm}$ long axes. The variation in shape reduces the number of amyloplasts exposed to the HGMF if the wedge is positioned $3 \mathrm{~mm}$ below the tip to less than a quarter of the cross-sectional area. When the wedge is positioned at the tip two extreme situations are possible such that the longer axis of the elliptical crosssection is parallel or perpendicular to the wedge edge (Fig. 2). In the parallel position about half of the crosssection of the coleoptile is subjected to the effective HGMF versus about a quarter in the perpendicular position. While the data presented so far were obtained from coleoptiles aligned parallel to the wedge, the influence of the volume of plant tissue subjected to the effective HGMF was studied as well. Parallel orientation of wedge and long axis of the coleoptile tip resulted in a higher percentage of curvature than perpendicular orientation (Fig. 9). These data suggest that curvature is correlated with the proportion of statocytes subjected to the HGMF that approximates gravity effects. Despite the geometric differences of the area exposed to the HGMF, these studies suggest that amyloplast containing cells vary in their sensitivity based upon their longitudinal position within the coleoptile. The curvature in coleoptiles that were positioned with their tip perpendicular to the wedge was greater than the curvature of coleoptiles that were exposed to an effective HGMF $1 \mathrm{~mm}$ and $3 \mathrm{~mm}$ below the tip, although the cross-sectional area of tissue of the coleoptile exposed to an effective HGMF was similar. If amyloplasts in shoots are confined to a distinct area or tissue, for example, cereal pulvini (Song et al., 1988; Brock et al., 1989; Brock and Kaufman, 1990) it should be possible to influence this area of the plant by a HGMF with a sufficient dynamic factor and to induce curvature away from the HGMF.

These results contrast with those obtained by Audus and Whish (1964) and Schwarzacher and Audus (1973). These authors used considerably weaker gradients, reported curvature of shoots and roots away from the higher field density, and could not verify amyloplast displacement. Therefore, it is possible that weaker gradients than those employed in the previous (Kuznetsov and Hasenstein, 1996) and present studies may cause qualitatively different curvature of shoots due to effects other than amyloplasts displacement.

These results (Kuznetsov and Hasenstein, 1996, and 
Table 1. Growth rate and curvature of vertically and horizontally oriented barley coleoptiles during $3.5 \mathrm{~h}$ after removal of various lengths of apical tissue (values $\pm S E$ )

\begin{tabular}{|c|c|c|c|c|c|}
\hline \multirow{2}{*}{$\begin{array}{l}\text { Length of apical } \\
\text { tissue removed } \\
(\mathrm{mm})\end{array}$} & \multicolumn{2}{|c|}{ Vertical orientation } & \multicolumn{3}{|c|}{ Horizontal orientation } \\
\hline & $\begin{array}{l}\text { Growth rate } \\
\left(\mathrm{mm} \mathrm{h}^{-1}\right)\end{array}$ & $n$ & $\begin{array}{l}\text { Growth rate } \\
\left(\mathrm{mm} \mathrm{h}^{-1}\right)\end{array}$ & $\begin{array}{l}\text { Curvature rate } \\
\left(\operatorname{deg} \mathrm{h}^{-1}\right)\end{array}$ & $n$ \\
\hline 0.0 & $2.92 \pm 0.35$ & 20 & $0.84 \pm 0.09$ & $26.3 \pm 1.3$ & 21 \\
\hline 0.3 & $2.53 \pm 0.27$ & 23 & $0.77 \pm 0.07$ & $21.1 \pm 1.7$ & 25 \\
\hline 1.0 & $2.00 \pm 0.17$ & 29 & $0.56 \pm 0.07$ & $17.6 \pm 1.4$ & 26 \\
\hline
\end{tabular}

this report) indicate that a HGMF induces a ponderomotive magnetic force that acts on starch-filled amyloplasts. The HGMF can thus simulate gravity or accelerative forces with the advantage that it can be confined to small areas. The displacement of amyloplasts inside the cells due to this ponderomotive force causes negatively gravitropic coleoptiles to curve toward, and plant roots, which typically show positive gravitropism, to curve away from the stronger field area. The experiments strongly support the concept of statolith-based gravity sensing because all tested differences in geometry of the HGMF and controls demonstrate that not the magnetic field per se but the amyloplast-specific ponderomotive force causes curvature.

\section{Acknowledgement}

This research was supported by NASA grants NAGW-3565 and NAG10-0190.

\section{References}

Audus LJ. 1960. Magnetotropism: a new plant growth response. Nature 185, 132-4.

Audus LJ, Whish JC. 1964. Magnetotropism. In: Barnothy MF, ed. Biological effects of magnetic fields, Vol 1. New York: Plenum Press, 170-82.

Braam J, Davis RW. 1990. Rain-, wind- and touch-induced expression of calmodulin and calmodulin related genes in Arabidopsis. Cell 60, 357-64.

Brock TG, Kaufman PB. 1990. Movement in grass shoots. In: Satter RI, Gorton HL, Vogelmann TC, eds. The pulvinus: motor organ for leaf movement. 59-71.

Brock TG, Lu CR, Ghosen NS, Kaufman PB. 1989. Localization and pattern of graviresponse across the pulvinus of barley Hordeum vulgare. Plant Physiology 91, 744-8.

Conover WJ. 1971. Practical non-parametric statistics. New York: John Wiley \& Sons Inc.

Digby J, Firn RD. 1995. The gravitropic set-point angle (GSA): the identification of an important developmentally controlled variable governing plant architecture. Plant, Cell and Environment. 18, 1434-40.

Hasenstein KH, Evans ML, Stinemetz LC, Moore R, Fondren WM, Koon EC, Higby MA, Smucker AJM. 1988. Comparative effectiveness of metal ions in inducing curvature of primary roots of Zea mays. Plant Physiology 86, 885-9.

Jaffe MJ. 1973. Thigmomorphogenesis: the response of plant growth and development to mechanical stimulation. Planta 114, 143-57.

Kuznetsov OA, Hasenstein KH. 1996. Magnetophoretic induction of root curvature. Planta 198, 87-94.

Kuznetsov AA, Kuznetsov OA. 1989. Simulation of gravity force for plants by high gradient magnetic field. Biofizika 35, 835-40.

Piruzyan LA, Kuznetsov AA, Chikov VM. 1980. About the magnetic heterogeneity of biological systems. Izvestiya Academia Science USSR, Biological Series 5, 645-53.

Schwarzacher JC, Audus LJ. 1973. Further studies in magnetotropism. Journal of Experimental Botany 24, 459-74.

Song I, Lu CR, Brock TG, Kaufman PB. 1988. Do starch statoliths act as the gravisensors in cereal grass pulvini? Plant Physiology 86, 1155-62.

SysStat Software. 1992. SYSTAT for windows. Statistics, Version 5. Evanstown, IL, USA: SysStat Inc. 